\title{
ON IMMERSIONS OF $k$-CONNECTED $n$-MANIFOLDS
}

\author{
B. H. LI AND M. E. MAHOWALD
}

(Communicated by Frederick R. Cohen)

\begin{abstract}
In this note we classify up to regular homotopy the classes of immersions of $k$-connected closed differentiable $n$-manifolds in $\mathbb{R}^{2 n-k}$.
\end{abstract}

In [W] it was claimed that for a $k$-connected closed differentiable manifold $M$ of dimension $n$ with $0 \leq 2 k \leq n-2$, the regular homotopy classes of $M$ in $\mathbb{R}^{2 n-k}$ are in one to one correspondence with $\pi_{n}\left(V_{2 n-k, n}\right)$, where $V_{2 n-k, n}$ is a Stiefel manifold. But this result is incorrect. In this note, we will present the correct answer and give counterexamples to the claim of [W].

To state our result, some definitions are needed. First, let $\pi_{i}(M)$ be the $i$ th homotopy group of $M$. Then

$$
h: \pi_{k+1}(M) \rightarrow \widetilde{K O}\left(S^{k+1}\right)
$$

is defined as follows: If $\alpha \in \pi_{k+1}(M)$ is represented by a map $\tilde{\alpha}: S^{k+1} \rightarrow M$, then $h(\alpha)$ is the element in $\widetilde{K O}\left(S^{k+1}\right)$ represented by $2 \tilde{\alpha}^{*} \nu_{M}$, where $\nu_{M}$ is the stable normal bundle of $M$. It is easy to see that $h$ is well defined and is a homomorphism.

Next, let

$$
k: \widetilde{K O}\left(S^{k+1}\right) \rightarrow \pi_{k}(S O)
$$

be the natural isomorphism,

$$
J: \pi_{k}(S O) \rightarrow \pi_{k}^{s}=\pi_{n}\left(S^{n-k}\right)
$$

be the $J$-homomorphism, and let

$$
S^{n-k} \stackrel{i}{\rightarrow} V_{2 n-k, n} \rightarrow V_{2 n-k, n-1}
$$

be the natural fibration. Let

$$
e=i_{*} \circ J \circ k \circ h: \pi_{k+1}(M) \rightarrow \pi_{n}\left(V_{2 n-k, n}\right) .
$$

Then we have

Received by the editors August 23, 1991.

1991 Mathematics Subject Classification. Primary 57N35.

The work of the first author was supported at MRSI by NSF Grant \#DMS 8505550 and the second author was supported by the NSF. 
Theorem. If $0 \leq 2 k \leq n-2$ and $M$ is a $k$-connected closed differentiable manifold, then the regular homotopy classes of immersions of $M$ in $\mathbb{R}^{2 n-k}$ are in one-to-one correspondence with the cokernel of $e$.

Example. Let $\mathbb{K}^{2}$ be the Cayley projective plane and $M=\mathbb{K}^{2} \times S^{23}$. Then $M$ is 7-connected with dimension 39 , and the homotopy sequence of the fibration $S^{32} \rightarrow V_{71,39} \rightarrow V_{71,38}$ gives the split exact sequence

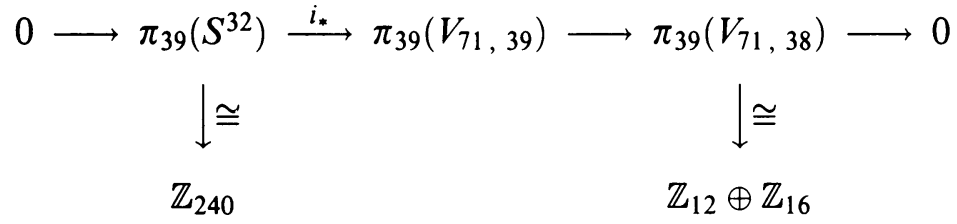

Now, the tangent bundle of $\mathbb{K}^{2}$ restricted to $\mathbb{K}^{1} \cong S^{8}$ is stably equivalent to the canonical 8-dimensional vector bundle, which can be defined by regarding $S^{8} \cong \mathbb{R}^{8} \cup\{\infty\}=D_{+} \cup D_{-}$and $\mathbb{R}^{8}$ as the Cayley algebra. Let $D_{+}=\{x \in$ $\left.\mathbb{R}^{8} /\|x\| \leq 1\right\}$ and $D_{-}=S^{8}-D_{+}$. Glue $D_{+} \times \mathbb{R}^{8}$ and $D_{-} \times \mathbb{R}^{8}$ over $D_{+} \cap D_{-}=S^{7}$ by $(x, y) \sim(x, x y)$ where $x y$ is the product of $x \in S^{7}$ and $y \in \mathbb{R}^{8}$ as Cayley numbers (see Steenrod [S, p. 109]).

Let $1, e_{1}, \ldots, e_{7}$ be the units of Cayley algebra. Then $x \rightarrow\left(x, x e_{1}, \ldots\right.$, $\left.x e_{7}\right)$ defines a map $S^{7} \rightarrow S O(8)$, which represents a generator of the first summand of $\pi_{7}(S O(8)) \cong \pi_{7}\left(S^{7}\right) \oplus \pi_{7}(S O(7)) \cong \mathbb{Z} \oplus \mathbb{Z}$, hence a generator of $\pi_{7}(S O)$ (cf. [L, Remark 4]). Thus $\left.2 T(M)\right|_{\mathrm{K}^{1}}$ represents twice a generator of $\widetilde{K O}\left(S^{8}\right)$. Since $J=\pi_{7}(S O) \rightarrow \pi_{7}^{S}$ is surjective, we see that the cokernel of $e$ is isomorphic to $\mathbb{Z}_{2} \oplus \mathbb{Z}_{12} \oplus \mathbb{Z}_{16}$. This shows that the result of [W] is incorrect.

We can produce more examples. Let $\xi$ be any 8-dimensional vector bundle over $S^{8}$. Then the second Pontriagin class $p_{2}(\xi)=6 q$ for some $q \in \mathbb{Z}$ (cf. [K]). Let $M=S(\xi \oplus 1) \times S^{23}$, where $S(\xi \oplus 1)$ is the total space of the sphere bundle of $(\xi \oplus 1)$. Then $\pi_{8}(M)=Z \oplus Z$ and $h$ maps one summand to 0 , another to $2 q \mathbb{Z}$. Thus the image of $e$ can be any subgroup of $\pi_{7}^{S} \cong \mathbb{Z}_{240}$ consisting of even elements, and only when $q \equiv 0 \bmod 120$ is the result of [W] true.

Since $2 \widetilde{K O}\left(S^{k+1}\right)=0$, if $k \not \equiv 3,7 \bmod 8$, we have

Corollary 1. If $k \not \equiv 3,7 \bmod 8$, then the cokernel of $e$ is $\pi\left(V_{2 n-k, n}\right)$.

If $n-k$ is odd, then $i_{*}=\pi_{n}\left(S^{n-k}\right) \rightarrow \pi_{n}\left(V_{2 n-k, n}\right)$ sends $2 \pi_{n}\left(S^{n-k}\right)$ to zero since the first two essential cells of the Stiefel manifold form a $Z / 2$ Moore space. So we have

Corollary 2. If $n-k$ is odd and $k \not \equiv 3,7 \bmod 8$, then the image of $e$ is zero. Proof of the theorem. By using normal bordism theory, we see from [Ko] or [D] that $\operatorname{Imm}\left[M, \mathbb{R}^{2 n-k}\right]\left(\right.$ or $\left.\left[M \propto \mathbb{R}^{2 n-k}\right]\right) \cong \Omega_{k}\left(M \times P^{\infty}, \phi\right)$, where $P^{\infty}$ is the infinite real projective space, $\phi=(2 n-k) \lambda-\lambda \otimes T(M)-T(M)$, and $\lambda$ is the canonical line bundle over $P^{\infty}$. There is an exact sequence

$$
\begin{aligned}
& \Omega_{k+1}\left(M \times P^{\infty}, * \times P^{\infty}, \phi\right) \stackrel{\partial}{\rightarrow} \Omega_{k}\left(P^{\infty},\left.\phi\right|_{P^{\infty}}\right) \\
& \quad \rightarrow \Omega_{k}\left(M \times P^{\infty}, \phi\right) \rightarrow \Omega_{k}\left(M \times P^{\infty}, * \times P^{\infty}, \phi\right)
\end{aligned}
$$

(cf. [D, p. 310]), where $* \in M$ is a point. Since $\left(M \times P^{\infty}, P^{\infty}\right)$ is $k$-connected, it follows from Proposition 5.1 in [D] that $\Omega_{k}\left(M \times P^{\infty}, * \times P^{\infty}, \phi\right)=0$, 
and the natural homomorphism

$$
\mu: \Omega_{k+1}\left(M \times P^{\infty}, * \times P^{\infty}, \phi\right) \rightarrow H_{k+1}\left(M \times P^{\infty}, * \times P^{\infty}, Z(\phi)\right)
$$

is an isomorphism.

Now look at the exact sequence

$$
\begin{aligned}
\rightarrow H_{k+1}\left(P^{\infty}, 2\left(\left.\phi\right|_{P \infty}\right)\right) & \stackrel{i_{k+1}}{\rightarrow} H_{k+1}\left(M \times P^{\infty}, z(\phi)\right) \\
& \stackrel{j}{\rightarrow} H_{k+1}\left(M \times P^{\infty}, * \times P^{\infty}, z(\phi)\right) \\
& \stackrel{\partial}{\rightarrow} H_{k}\left(P^{\infty}, z\left(\left.\phi\right|_{P \infty}\right)\right) \stackrel{i_{k}}{\rightarrow} H_{k}\left(M \times P^{\infty}, z(\phi)\right) .
\end{aligned}
$$

Since $W_{1}(\phi)=W_{1}((n-k) \lambda)$, we have $Z(\phi)=Z \otimes Z((n-k) \lambda)$. Hence, by the Künneth formula for twisted coefficients,

$$
\begin{aligned}
& H_{*}\left(M \times P^{\infty}, Z(\phi)\right) \\
& \quad=H_{*}\left(P^{\infty}, Z((n-k) \lambda)\right) \oplus H_{*}(M, Z) \otimes H_{0}\left(P^{\infty}, Z((n-k) \lambda)\right)
\end{aligned}
$$

for $* \leq k+1$. This shows that $i_{k}$ is an isomorphism and $j$ is an isomorphism from $H_{k+1}(M, Z) \otimes H_{0}\left(P^{\infty}, \mathbb{Z}((n-k) \lambda)\right)$ to $H_{k+1}\left(M \times P^{\infty}, P^{\infty}, Z(\phi)\right)$.

Now $\Omega_{k}\left(P^{\infty},\left.\phi\right|_{P \infty}\right)=\Omega_{k}\left(P^{\infty},(n-k) \lambda\right)=\pi_{n}\left(V_{2 n-k, n}\right)$ (cf. [D, Proposition 7.3] or [Ko, Proposition 5.4]). We need only to calculate the image of $\partial$ in the following diagrams:

$$
\begin{aligned}
& \Omega_{k+1}\left(M \times P^{\infty}, P^{\infty}, \phi\right) \\
& \stackrel{\partial}{\longrightarrow} \Omega_{k}\left(P^{\infty},\left.\phi\right|_{P \infty}\right) \\
& \downarrow \cong \\
& \downarrow \cong \\
& H_{k+1}(M, Z) \otimes H_{0}\left(P^{\infty}, z((n-k) \lambda)\right) \\
& \pi_{n}\left(V_{2 n-k, n}\right)
\end{aligned}
$$

By the Hurewicz isomorphism theorem,

$$
\begin{aligned}
H_{k+1}(M) & \cong H_{k+1}(M, \mathbb{Z}) \text { if } k>0, \\
\frac{\pi_{1}(M)}{\left[\pi_{1}(M), \pi_{1}(M)\right]} \cong H_{1}(M, \mathbb{Z}) & \text { if } k=0 .
\end{aligned}
$$

Let $S^{k+1}=D_{+} \cup D_{-}$, where $D_{+}$and $D_{-}$are disks with $D_{+} \cap D_{-}=S^{k}$, and $c: S^{k+1} \rightarrow P^{\infty}$ be a constant map. For any $\alpha \in \pi_{k+1}(M, *)$, we can choose $\tilde{\alpha}: S^{k+1} \rightarrow M$, representing $\alpha$ with $\tilde{\alpha}\left(D_{-}\right)=*$.

Let $\bar{\alpha}=(\tilde{\alpha}, c): D_{+} \rightarrow M \times P^{\infty}$. Then $\bar{\alpha}$ maps $\left(D_{+}, \partial D_{+}\right)$into $(M \times$ $\left.P^{\infty}, * \times P^{\infty}\right)$. Regard $\phi$ as a stable bundle; then $T\left(D_{+}\right) \oplus \bar{\alpha}^{*} \phi$ obviously has a trivialization $V$. Thus the triple $\left(\left(D_{+}, \partial D_{+}\right), \bar{\alpha}, V\right)$ defines an element in $\Omega_{k+1}\left(M \times P^{\infty}, * \times P^{\infty}, \phi\right)$, and $\left(\partial D_{+},\left.\bar{\alpha}\right|_{\partial D_{+}},\left.V\right|_{\partial D_{+}}\right)$defines an element in $\Omega_{k}(*$, trivial $) \equiv \pi_{k}^{s}$, which can be regarded as an element of $\Omega_{k}\left(P^{\infty},\left.\phi\right|_{P^{\infty}}\right.$ via the inclusion $*=c\left(S^{k+1}\right) \subset P^{\infty}$. From the isomorphisms

$$
\begin{aligned}
\Omega_{k+1}\left(M \times P^{\infty}, * \times P^{\infty}, \phi\right) & \cong H_{k+1}\left(M \times P^{\infty}, * \times P^{\infty}, Z(\phi)\right) \\
& \cong H_{k+1}(M, Z(\phi)) \otimes H_{0}\left(P^{\infty}, Z((n-k) \lambda)\right) \\
& \cong \pi_{k+1}(M) \otimes H_{0}\left(P^{\infty}, Z((n-k) \lambda) .\right.
\end{aligned}
$$

We see that the image of

$$
\partial: \Omega_{k+1}\left(M \times P^{\infty}, * \times P^{\infty}, \phi\right) \rightarrow \Omega_{k}\left(P^{\infty},\left.\phi\right|_{P \infty}\right)
$$

is exactly the set $\left\{\left[\left(\partial D_{+},\left.\bar{\alpha}\right|_{\partial D_{+}},\left.V\right|_{\partial D_{+}}\right)\right] / \alpha \in \pi_{k+1}(M)\right\}$. 
Regard $\left.V\right|_{\partial D_{+}}$as a trivialization of

$$
\left.\left(T\left(D_{-}\right) \oplus \tilde{\alpha}^{*} \phi\right)\right|_{\partial D_{-}}
$$

then it defines an element of $\pi_{k}(S O)$, which is the value of $k: \widetilde{K O}\left(S^{k+1}\right) \rightarrow$ $\pi_{k}(S O)$ on the stable bundle $\tilde{\alpha}^{*}\left(2 \nu_{M}\right)=h(\alpha)$. It is obvious that the element in $\Omega_{k}\left(*\right.$, trivial) defined by $\left(\partial D_{+},\left.\tilde{\alpha}\right|_{\partial D_{+}},\left.V\right|_{\partial D_{+}}\right)$is $(J \circ k \circ h)(\alpha)$.

Now, the only remaining thing is to see that the diagram

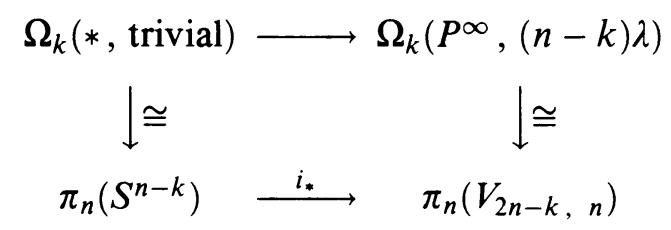

commutes. First

$$
\Omega_{k}\left(P^{\infty},(n-k) \lambda\right) \cong \Omega_{k}\left(P^{m-1},(n-k) \lambda\right) \cong \pi_{n}\left(\frac{P^{2 n-k-1}}{P^{n-k-1}}\right)
$$

where $P^{2 n-k-1} / P^{n-k-1}$ is the Thom space of $(n-k) \lambda$. Under the natural map

$$
P^{2 n-k-1} / P^{n-k-1} \rightarrow V_{2 n-k, n}
$$

the Thom space of $(n-k) \lambda$ restricted to a point maps onto $i\left(S^{n-k}\right) \subset V_{2 n-k, n}$. So the above diagram commutes. So far we have proved that in the following diagram

$$
\begin{array}{cc}
\Omega_{k+1}\left(M \times P^{\infty}, * \times P^{\infty}, \phi\right) & \stackrel{\partial}{\longrightarrow} \\
\Omega_{k}\left(P^{\infty},\left.\phi\right|_{P \infty}\right) \rightarrow \Omega_{k}\left(M \times P^{\infty}, \phi\right) \rightarrow 0 \\
\\
\pi_{k+1}(M) & \stackrel{e=i_{*} \circ J \circ k \circ h}{\longrightarrow} \pi_{n}\left(V_{2 n-k, n}\right)
\end{array}
$$

the images of $\partial$ and $e$ are isomorphic and hence so is the theorem.

\section{REFERENCES}

[D] J. P. Dax, Étude homotopique des espaces de plungements, Ann. Sci. École Norm. Sup. (4) 5 (1972), 303-377.

[K] M. Kervaire, A note on obstructions and characteristic classes, Amer. J. Math. 81 (1959), 773-784.

[Ko] U. Koschorke, Vector fields and other vector bundle morphisms-A singularity approach, Lecture Notes in Math, vol. 847, Springer-Verlag, 1981.

[L] B. H. Li, Parallelizability of algebraic knots and canonical framings, Sci. Sinica 27 (1984), 1164-1171.

[S] N. Steenrod, The topology of fibre bundles, Princeton Univ. Press, Princeton, NJ, 1951.

[W] R. Wiegmann, Immersions of highly connected manifold, Proc. Amer. Math. Soc. 90 (1984), 483-484. CHINA

Insitute of Systems Science, Academia Sinica, Beijing 100080, People's Republic of

Department of Mathematics, Northwestern University, Evanston, Illinois 60201

E-mail address: mark@math.nwu.edu 